\title{
FOLHAS DE ALFACE LISA (Lactuca sativa) MINIMAMENTE PROCESSADAS ARMAZENADAS EM ATMOSFERA MODIFICADA: ANÁLISES FÍSICAS, QUÍMICAS E FÍSICO-QUÍMICAS ${ }^{1}$
}

\author{
Ellen M S MENEZES ${ }^{2, *}$, Érica C. FERNANDES ${ }^{2}$, Armando U. O. SABAA-SRUR ${ }^{2}$
}

\begin{abstract}
RESUMO
Alfaces (Lactuca sativa) cv. lisa, após seleção e pré-lavagem, foram lavadas com água clorada a $5^{\circ} \mathrm{C}$ contendo 150 ppm de cloro residual livre e pH ajustado para 6.0, enxagüadas e centrifugadas por 8 minutos a 2200rpm. Em seguida, foram acondicionadas em embalagens plásticas de nylon poli 5 camadas, sem e com atmosfera modificada $\left(\mathrm{N}_{2}\right.$ contendo 20ppm de $\mathrm{O}_{2}$ e no máximo 20ppm umidade) e estocadas a $4^{\circ} \mathrm{C}$. Durante 5 dias foram realizadas mensurações de $\mathrm{pH}$, concentração de sólidos solúveis (graus Brix) e análises de acidez (mL NaOH $0,1 \mathrm{M} / 100 \mathrm{~g}$ folhas de alface). Os produtos sofreram acidificação detectável pela diminuição do pH em ambas as atmosferas. O incremento da acidez foi mostrado, sendo os registros maiores para as amostras controle (sem atmosfera modificada), e não ocorreram variações de graus Brix. Os resultados fizeram concluir que avanços tecnológicos vêm sendo alcançados visando melhorar a qualidade e vida-deprateleira do vegetal.
\end{abstract}

Palavras-chave: alface; vegetais minimamente processados; atmosfera modificada.

\section{SUMMARY}

MINIMALLY PROCESSED SMOOTH LETTUCE LEAVES (Lactuca sativa) STORED AT MODIFIED ATMOSPHERE: PHYSICAL, CHEMICAL AND PHYSICO-CHEMICAL ANALYSIS. Lettuce (Lactuca sativa) cv. smooth, after being selected and washed, was cleaned with chlorine water at $5^{\circ} \mathrm{C}$, with $150 \mathrm{ppm}$ of free chlorine and $\mathrm{pH}$ adjusted to 6.0 , rinsed and centrifuged for 8 minutes at $2200 \mathrm{rpm}$. Right after, the lettuce leaves were packaged in plastic bags made of nylon poli 5 layers, with and without modified atmosphere $\left(\mathrm{N}_{2}\right.$ having $20 \mathrm{ppm}$ of $\mathrm{O}_{2}$ and $20 \mathrm{ppm}$ of maximum moisture) and stored at $4^{\circ} \mathrm{C}$. During 5 days there were $\mathrm{pH}$, soluble solids concentration measurements and acid analysis ( $\mathrm{mL} \mathrm{NaOH} 0,1 \mathrm{M} / 100 \mathrm{~g}$ lettuce leaves). The products became acid, detectable by a pH decrease in both atmospheres. A raise in acidity was shown, being the highest marks for the control samples (without modified atmosphere) and there was no soluble solids variation. Results have shown that technological progress is being reached aiming to improve the vegetable's quality and shelf life. Keywords: lettuce; minimally processed vegetables; modified atmosphere.

\section{1 - INTRODUÇÃO}

Há uma tendência para o uso de alimentos cada vez mais naturais, valorizando o sabor original dos produtos, onde o consumidor prima pela qualidade, principalmente às relacionadas com o valor nutritivo, as condições higiênico-sanitárias e as características sensoriais, portanto novas tecnologias estão sendo desenvolvidas no processamento de alimentos [10]. Na década de 70 surgiram nos EUA vegetais pré-processados, reunindo a praticidade e a conveniência. Esse mercado se expandiu e continua crescendo em todo mundo, principalmente nos países desenvolvidos, justamente para atender a demanda mundial de vegetais frescos Minimamente Processados (VMP) ou "prontos para o consumo" [2]. Skura $\&$ Powrie estimaram que, no ano de 1995, mais da metade dos dólares gastos em lojas de conveniência com a compra de alimentos, foram voltados para a aquisição de itens prontos para o consumo, dentre eles, VMPs [8].

Os VMP englobam todos aqueles produtos frescos submetidos a pequeno processamento, normalmente, lavagem, corte, fatiamento, acabamento ou seleção e empacotamento [3, 12]. Alguns processadores substi-

1. Recebido para publicação em 23/04/2003. Aceito para publicação em 26/11/2004 (001101).

2. Depto. de Nutrição Básica e Experimental, Instituto de Nutrição Josué de Castro, Universidade Federal do Rio de Janeiro (UFRJ).

* A quem a correspondência deve ser enviada. tuem a atmosfera normal por modificada ou controlada e outros submetem os produtos a baixos níveis de irradiação visando manter o frescor e o estado natural dos produtos $[9,10]$. A embalagem com atmosfera modificada (AM) em combinação com a refrigeração constituem uma crescente técnica de preservação de VMP [4] visando manter os produtos em atmosfera com reduzido teor de $\mathrm{O}_{2}$ e enriquecida com $\mathrm{CO}_{2}$ [1], minimizando a deterioração do VMP. Os principais gases utilizados nas AMs são $\mathrm{O}_{2}, \mathrm{~N}_{2}$ e $\mathrm{CO}_{2}$ [11]. A escolha é influenciada pela flora microbiana capaz de crescer no produto, a sensibilidade do produto ao $\mathrm{O}_{2}$ e $\mathrm{CO}_{2}$ e a estabilidade da coloração. $\mathrm{O}$ $\mathrm{N}_{2}$ pode ser ainda utilizado com o intuito de promover atmosfera com baixas concentrações de $\mathrm{O}_{2}$, prevenindo o escurecimento enzimático indesejável do vegetal [4]. Muitos fatores influenciam a qualidade de alfaces MP incluindo práticas culturais, cultivar e maturação na colheita, métodos de colheita, manuseio do campo até o local de preparação, critério de inspeção, duração e condições de estocagem. Somente um sistema de manuseio integrado e completo permite um excelente controle sobre os fatores de qualidade [12]. Entre as hortaliças mais vendidas para o consumo in natura está a alface (Lactuca sativa), muito utilizada no consumo doméstico, nas cadeias de fast foods e restaurantes comerciais [7, 11 ], por isso o objetivo do estudo foi avaliar a eficiência do processamento mínimo de folhas de alface de maneira que as tornassem mais seguras ao consumo mantendo sua boa qualidade quanto às características fisicas, químicas e físico-químicas. 


\section{2 - MATERIAL E MÉTODOS}

Alface (Lactuca sativa) cv. Lisa, cultivada em estabelecimento agrícola, situado a $50 \mathrm{~km}$ do local foi colhido pela manhã, imediatamente transportado para o LAPAL/ IN. Cuidadosamente selecionada, onde foram retiradas as partes amassadas, murchas, marcadas e, danificadas por insetos ou dano físico, foram manualmente desfolhadas e lavadas com água corrente potável, para eliminação das sujidades e insetos.

As folhas foram novamente lavadas e desinfetadas sob imersão em água potável a $5^{\circ} \mathrm{C}$, contendo $150 \mathrm{ppm}$ de cloro residual livre (CRL) e o $\mathrm{pH}$ ajustado para 6.0 com ácido clorídrico (HCl). Após 10-15 minutos, fez-se o enxágüe em água potável declorada, seguida da centrifugação a $2.200 \mathrm{rpm}$ por $8 \mathrm{~min}$. Cerca de $20 \mathrm{~g}$ de alface eram acondicionados na embalagem (sacos de nylon poli 5 camadas) e selada. As embalagens foram divididas em higienizadas sem atmosfera modificada (HSAM) e higienizadas com atmosfera modificada à base de $\mathrm{N}_{2}$ contendo $20 \mathrm{ppm}$ de $\mathrm{O}_{2}$ e no máximo 20ppm umidade (HCAM). As embalagens armazenadas sob refrigeração $\left(4^{\circ} \mathrm{C}\right)$ durante 5 dias foram avaliadas diariamente em triplicatas através de análises físicas, químicas e físico-químicas.

Fez-se mensurações de pH e concentração de sólidos solúveis expressa em graus Brix através do potenciômetro e refratômetro de Abbe, respectivamente, e as análises de acidez expressas em $\mathrm{mL} \mathrm{NaOH} \mathrm{0,1M/}$ $100 \mathrm{~g}$ de folhas de alface [5].

\section{3 - RESULTADOS E DISCUSSÃO}

Além da composição química, os aspectos físicoquímicos de alfaces são bastante favoráveis ao desenvolvimento de microrganismos. $\mathrm{O} \mathrm{pH}$ dos tecidos vegetais situa-se entre 5-7, faixa bastante adequada para crescimento de bactérias e fungos [6]. Na Figura 1, observa-se a acidificação dos produtos submetidos às duas atmosferas, com discreta diminuição do $\mathrm{pH}$, porém sem grandes diferenças significativas entre amostras HCAM e as controles (HSAM), sendo creditado a amostra controle 3 (HSAM) o menor valor para o $\mathrm{pH}$. A presença de $\mathrm{O}_{2}$, como gás predominante dessa embalagem, tornouse o indutor de reações enzimáticas [6], fazendo com que houvesse o escurecimento e redução da qualidade desse vegetal.

A Figura 2 mostra um incremento da acidez no decorrer do período de armazenamento, sendo os maiores registros as amostras controles (HSAM), com uma acidez inicial média (TO) de $8,24 \mathrm{~mL} \mathrm{NaOH} 0,1 \mathrm{M} / 100 \mathrm{~g}$ de folhas, e final (T5) de $11,21 \mathrm{~mL} \mathrm{NaOH} 0,1 \mathrm{M} / 100 \mathrm{~g}$ de folhas, contra uma acidez inicial média (T0) de $7,13 \mathrm{~mL}$ $\mathrm{NaOH} 0,1 \mathrm{M} / 100 \mathrm{~g}$ de folhas, e final (T5) de $8,93 \mathrm{~mL} \mathrm{NaOH}$ $0,1 \mathrm{M} / 100 \mathrm{~g}$ de folhas nas amostras HCAM. Em uma das amostras (HSAM 1), o aumento foi evidente, provavelmente em função de falhas no processo ou durante o fechamento da embalagem.

O sabor dos vegetais frescos resulta da combinação de compostos voláteis com açúcar e ácidos [12]. A análise da concentração de sólidos solúveis se faz importante uma vez que os carboidratos, que também conferem sabor doce ao vegetal, são fontes energéticas para o crescimento de microrganismos. Então, o consumo desses açúcares pelos microrganismos pode ser medido pelos graus Brix, ou conforme SHEWFELT [12] propõe, pela razão Brix/acidez. Na Tabela 1, não houve variações significativas da concentração dos sólidos solúveis, mostrando a eficácia dos processos aplicados em relação ao crescimento de microrganismos.

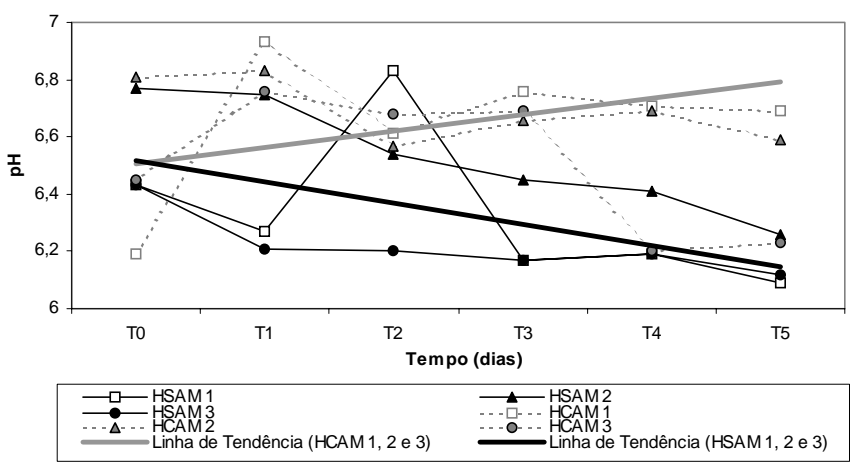

HSAM 1 (amostra 1 higienizada sem atmosfera modificada), HSAM 2 (amostra 2 higienizada sem atmosfera modificada) HSAM 3 (amostra 3 higienizada sem atmosfera modificada), HCAM 1 (amostra 1 higienizada com atmosfera modificada), HCAM 2 (amostra 2 higienizada com atmosfera modificada) e HCAM 3 (amostra 3 higienizada com atmosfera modificada).

FIGURA 1. Análises de $\mathrm{pH}$ das amostras HSAM e HCAM.

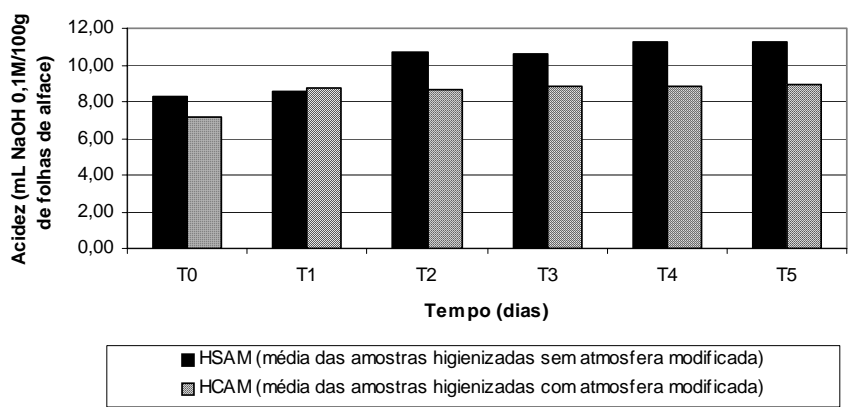

FIGURA 2. Médias da acidez das amostras (1, 2, e 3) HSAM e HCAM.

TABELA 1. Graus Brix (sólidos solúveis) das amostras (1, 2 e 3) HSAM e HCAM.

\begin{tabular}{ccccccc}
\hline Amostras & T0 & T1 & T2 & T3 & T4 & T5 \\
\hline HSAM 1 & 0,25 & 0,24 & 0,3 & 0,23 & 0,19 & 0,24 \\
HSAM 2 & 0,23 & 0,22 & 0,21 & 0,19 & 0,22 & 0,19 \\
HSAM 3 & 0,23 & 0,2 & 0,21 & 0,22 & 0,3 & 0,21 \\
\hline Médias & 0,24 & 0,22 & 0,24 & 0,22 & 0,23 & 0,22 \\
Desvio Padrão & 0,01 & 0,02 & 0,04 & 0,02 & 0,05 & 0,02 \\
& & & & & & \\
\hline HCAM 1 & 0,22 & 0,22 & 0,24 & 0,23 & 0,22 & 0,2 \\
HCAM 2 & 0,28 & 0,22 & 0,2 & 0,33 & 0,22 & 0,21 \\
HCAM 3 & 0,2 & 0,21 & 0,2 & 0,19 & 0,19 & 0,18 \\
\hline Médias & 0,23 & 0,22 & 0,22 & 0,24 & 0,22 & 0,22 \\
Desvio Padrão & 0,03 & 0,01 & 0,02 & 0,06 & 0,02 & 0,02 \\
\hline
\end{tabular}


O decréscimo na qualidade do vegetal está primeiramente baseado na aparência, então um VMP deve ter consistência firme, aparência de frescor, cor aceitável, e ser razoavelmente livre de defeitos [12]. A deterioração de alfaces é evidenciada pela perda de cor, escurecimento das bordas cortadas, e perda de turgidez [7]. Aos resultados da avaliação visual de textura e frescor dos VMPs, todas as embalagens, apresentavam bons aspectos por aproximadamente 2 semanas, porém as HCAM se estenderam mais que as HSAM.

\section{4 - CONCLUSÕES}

Avanços tecnológicos têm sido alcançados visando melhorar a qualidade e vida-de-prateleira de Vegetais Minimamente Processados (VMPs).

A lavagem e desinfecção têm mostrado, entre outros, como uma etapa benéfica importante na obtenção dos Vegetais Minimamente Processados (VMPs).

O processo de lavagem adotando o cloro como agente desinfectante mostrou-se capaz de beneficiar na manutenção de uma boa qualidade das hortaliças.

Os resultados das análises físico-químicas e químicas foram de representatividade para se diagnosticar diferenças entre os processamentos mínimos adotados na pesquisa.

A adoção de uma atmosfera modificada com nitrogênio, oxigênio $<20 \mathrm{ppm}$ e umidade $<20 \mathrm{ppm}$, mostrou-se também como um bom e benéfico processo de conservação dos vegetais.

\section{5 - REFERÊNCIAS BIBLIOGRÁFICAS}

[1] ARTÉS, F.; CASTAÑER, M.; GIL, M. I. .Revisión: E1 pardeamiento enzimático en frutas y hortalizas minimamente processadas. Food Science Technology International, v. 4, n. 6, p. 377-389, 1998.

[2] BERBARI, S.A.G.; PASCHOALINO, J. E.; SILVEIRA, N. F. A.. Efeito do cloro na água de lavagem para desinfecção de alface minimamente processada. Ciênc. Tecnol. Aliment., v. 21, n. 2, p. 197-201, 2001.

[3] CANTWELL, M. Food Safety: Microbiological Concerns.
Perishables Handling Newsletter Issue, v. 81, p. 15-16, 1995.

[4] FRANCIS, G.A.; O`BEIRNE, D. Effects of gas atmosphere, antimicrobiol dip and temperature on the fate of Listeria innocua and Listeria monocytogenes on minimally processed lettuce. International Journal of Food Science and Technology, v. 32, p. 141-151, 1997.

[5] INSTITUTO ADOLFO LUTZ. Normas Analiticas do Instituto Adolfo Lutz, métodos químicos e físicos de análise de alimentos. 3a edição. São Paulo, v. 1, 1985. 533p.

[6] JAY, J. Modern Food Microbiology. $4^{\text {th }}$ edition. Van Nostrand Reinhold: New York, 1992.

[7] KING JR., A.D.; MAgNUSON, J. A.; TÖRÖK, T.; GOODMAN, N.. Microbiol Flora and Storage Quality of Partially Processed Lettuce. Journal of Food Science, v. 56, n. 2, p. 459-462, 1991.

[8] LIMA, J.M.; MASSON, M.L. Alimentos minimamente processados: a nova tendência de mercado. Nutrição Brasil. Ano 2, n. 3, p. 154-159, 2003.

[9] LIMA, K.S.C. Qualidade em cenouras (Daucus carota L.) cv. Nantes Minimamente Processadas e irradiadas: Avaliação química, física e microbiológica. Seropédica, 2001, 149p. Dissertação (Mestre em Tecnologia de Alimentos), Universidade Federal Rural do Rio de Janeiro (UFRRJ).

[10] LIMA, K.S. LIMA, A. L. S.; LUCHESE, R. H.; GODOY, R. L. O.; SABAA-SRUR, A. U. O. Cenouras Minimamente processadas em embalagens com atmosfera modificadas e tratadas com radiação gama: avaliação microbiológica, físico-química e química. Ciênc. Tecnol. Aliment., v. 23, n. 2, p. 240-250, 2003.

[11] PHILLIPS, C.A. Review: Modified Atmosphere Packing and its effects on the microbiological quality and safety of produce. International Journal of Food Science and Technology, v. 31, p. 463-479, 1996.

[12] SHEWFELT, R.L. Quality of Minimally Processed Fruits and Vegetables. Journal of Food Quality, v. 10, p. 143-156, 1987.

\section{6 - AGRADECIMENTOS}

Os autores são gratos ao Conselho Nacional de Desenvolvimento Científico e Tecnológico (CNPq) pelo suporte financeiro capacitando a equipe a realizar pesquisas, e nos devotando credibilidade e incentivo para continuar desenvolvendo-as. 